\title{
Conservation Laws in Quantum-Correlation-Function Dynamics
}

\author{
Wei Wang ${ }^{1}$ and Mitsuo Takeda ${ }^{2}$ \\ ${ }^{1}$ Department of Mechanical Engineering, School of Engineering and Physical Sciences, Heriot-Watt University, \\ Edinburgh EH14 4AS, UK \\ ${ }^{2}$ Department of Engineering Science, Graduate School of Informatics and Engineering, The University of Electro-Communications, \\ 1-5-1, Chofugaoka, Chofu, Tokyo 182-8585, Japan
}

Correspondence should be addressed to Wei Wang, w.wang@hw.ac.uk

Received 22 November 2009; Accepted 31 March 2010

Academic Editor: Oleg V. Angelsky

Copyright $\odot 2010$ W. Wang and M. Takeda. This is an open access article distributed under the Creative Commons Attribution License, which permits unrestricted use, distribution, and reproduction in any medium, provided the original work is properly cited.

\begin{abstract}
For a complete and lucid discussion of quantum correlation, we introduced two new first-order correlation tensors defined as linear combinations of the general coherence tensors of the quantized fields and derived the associated coherence potentials governing the propagation of quantum correlation. On the basis of these quantum optical coherence tensors, we further introduced new concepts of scalar, vector and tensor densities and presented some related properties, such as conservation laws and the wave-particle duality for quantum correlation, which provide new insights into photon statistics and quantum correlation.
\end{abstract}

\section{Introduction}

Fields interact with atoms in a fundamentally random or stochastic way. As a consequence, statistical interpretation of the outcome of most optical experiments becomes indispensable due to the fact that any measurement of light is accompanied by certain unavoidable fluctuations $[1,2]$. Among various descriptions of the statistical properties of light, correlation between the fields at different space-time points, known as the correlation function, has long been recognized as the most fundamental physical quantity that plays a crucial role in photon correlations. The concept of optical correlation, first introduced by Wolf, has laid a foundation on which many important problems in classical statistical optics can be treated in a unified way. Also worth special mention is the quantum theory of optical coherence created by Glauber [3-5], who took a quantum electrodynamics approach to the problems of photon statistics. Because of its theoretical and practical importance, coherence theory and quantum optics have developed into a challenging multifarious field of research.

In quantum optics, detection of photons based on the absorption of photons via the photoelectric effect constitutes the basis of the measurements of the optical field. Photon statistics and quantum correlations have been studied extensively. However, the discussions in most of papers are restricted to the correlations between the same kinds of fields (either between electric fields $\mathbf{E}$ themselves or between magnetic fields $\mathbf{H}$ themselves) at different spacetime points, and the mixed correlation between electric field and magnetic field, introduced by Mehta and Wolf [6], seems to have received less attention in spite of its importance. Although the quantum theory for the correlations between the same kind of fields has already been established and is indeed informative, this alone cannot constitute a selfconsistent theoretical framework for the full description of the coherence properties of the quantum electromagnetic fields. The need for a more self-contained quantum correlation theory involving the $\mathbf{E H}$-mixed correlations has motivated us to take a fully quantum electrodynamics approach to the problems of quantum correlations.

In this paper, we will introduce, for the first time, two second-order correlation tensors: $\mathbb{E}_{j k}\left(\mathbf{x}_{1}, t_{1} ; \mathbf{x}_{2}, t_{2}\right)$ and $\mathbb{S}_{j k}\left(\mathbf{x}_{1}, t_{1} ; \mathbf{x}_{2}, t_{2}\right)$ for the complete and lucid description of quantum correlation on the basis of the quantized field theory. In formal analogy to the energy conservation, momentum conservation, and angular momentum laws for vector fields in classical electromagnetism, some new densities derived from $\mathbb{E}_{j k}$ and $\mathbb{S}_{j k}$ are introduced to quantum correlation theory and are related by new continuity 
equations, which indicate new conservation laws in quantum correlation theory. In terms of Fock states and coherent states, we will represent the newly introduced densities and reveal the nature of the wave-particle duality for the quantum correlation.

\section{Quantum Coherence Tensors}

To fully characterize the correlations involving all the components of the electromagnetic fields at any space-time points, more general coherence matrices are needed. In addition to Glauber's E-only correlation tensor [3-5], other first-order magnetic $\mathbf{H}$-correlation and $\mathbf{E H}$ mixed-correlation tensors have been defined by Mehta and Wolf [6], namely,

$$
\begin{aligned}
E_{j k}\left(\mathbf{x}_{1}, t_{1} ; \mathbf{x}_{2}, t_{2}\right) & =\operatorname{Tr}\left\{\hat{\rho} \hat{\mathbf{E}}_{j}^{(-)}\left(\mathbf{x}_{1}, t_{1}\right) \hat{\mathbf{E}}_{k}^{(+)}\left(\mathbf{x}_{2}, t_{2}\right)\right\}, \\
H_{j k}\left(\mathbf{x}_{1}, t_{1} ; \mathbf{x}_{2}, t_{2}\right) & =\operatorname{Tr}\left\{\hat{\rho} \hat{\mathbf{H}}_{j}^{(-)}\left(\mathbf{x}_{1}, t_{1}\right) \hat{\mathbf{H}}_{k}^{(+)}\left(\mathbf{x}_{2}, t_{2}\right)\right\}, \\
M_{j k}\left(\mathbf{x}_{1}, t_{1} ; \mathbf{x}_{2}, t_{2}\right) & =\operatorname{Tr}\left\{\hat{\rho} \widehat{\mathbf{E}}_{j}^{(-)}\left(\mathbf{x}_{1}, t_{1}\right) \hat{\mathbf{H}}_{k}^{(+)}\left(\mathbf{x}_{2}, t_{2}\right)\right\}, \\
N_{j k}\left(\mathbf{x}_{1}, t_{1} ; \mathbf{x}_{2}, t_{2}\right) & =\operatorname{Tr}\left\{\hat{\rho} \hat{\mathbf{H}}_{j}^{(-)}\left(\mathbf{x}_{1}, t_{1}\right) \hat{\mathbf{E}}_{k}^{(+)}\left(\mathbf{x}_{2}, t_{2}\right)\right\},
\end{aligned}
$$

where $\hat{\rho}$ is the density operator describing the field, the symbol $\operatorname{Tr}\{\cdots\}$ stands for the trace operation, the indices $(j, k=1,2,3)$ label Cartesian components: $(\hat{\mathbf{x}}, \hat{\mathbf{y}}, \hat{\mathbf{z}})$, and we have already separated the electromagnetic field operators into their positive-frequency parts $\widehat{\mathbf{E}}^{(+)}$and $\hat{\mathbf{H}}^{(+)}$and their negative-frequency parts $\widehat{\mathbf{E}}^{(-)}$and $\hat{\mathbf{H}}^{(-)}$, respectively.

Since the electric and magnetic field operators are related by Maxwell's equations in free space, their corresponding negative frequency operators $\widehat{\mathbf{E}}^{(-)}$and $\hat{\mathbf{H}}^{(-)}$can also be expressed in the tensor form as

$$
\begin{gathered}
\varepsilon_{j k l} \partial_{k}^{1} \widehat{\mathbf{E}}_{l}^{(-)}\left(\mathbf{x}_{1}, t_{1}\right)+\frac{1}{c} \frac{\partial}{\partial t_{1}} \hat{\mathbf{H}}_{j}^{(-)}\left(\mathbf{x}_{1}, t_{1}\right)=0, \\
\varepsilon_{j k l} \partial_{k}^{1} \hat{\mathbf{H}}_{l}^{(-)}\left(\mathbf{x}_{1}, t_{1}\right)-\frac{1}{c} \frac{\partial}{\partial t_{1}} \widehat{\mathbf{E}}_{j}^{(-)}\left(\mathbf{x}_{1}, t_{1}\right)=0, \\
\partial_{j}^{1} \hat{\mathbf{E}}_{j}^{(-)}\left(\mathbf{x}_{1}, t_{1}\right)=0, \\
\partial_{j}^{1} \hat{\mathbf{H}}_{j}^{(-)}\left(\mathbf{x}_{1}, t_{1}\right)=0
\end{gathered}
$$

where $c$ indicates the speed of light, $\varepsilon_{j k l}$ is the unit tensor of Levi-Civita with antisymmetry, $\partial_{j}^{1}$ and $\partial / \partial t_{1}$ are the differential operations to be performed with respect to the point $\mathbf{x}_{1}$ and $t_{1}$, and Einstein's summation convention has been employed. One of the essential aspects in which quantum field theory differs from classical theory is that two values of the field operators taken at different space-time points do not, in general, commute with one another. After multiplying both sides of (2) by $\hat{\mathbf{E}}_{m}^{(+)}\left(\mathbf{x}_{2}, t_{2}\right)$ and placing it under the operator signs, we obtain the relation

$$
\begin{aligned}
\varepsilon_{j k l} \partial_{k}^{1} \hat{\mathbf{E}}_{l}^{(-)}\left(\mathbf{x}_{1}, t_{1}\right) \widehat{\mathbf{E}}_{m}^{(+)}\left(\mathbf{x}_{2}, t_{2}\right) & \\
& +\frac{1}{c} \frac{\partial}{\partial t_{1}} \hat{\mathbf{H}}_{j}^{(-)}\left(\mathbf{x}_{1}, t_{1}\right) \hat{\mathbf{E}}_{m}^{(+)}\left(\mathbf{x}_{2}, t_{2}\right)=0 .
\end{aligned}
$$

If we take the quantum average of (6) and interchange the order of the quantum average and differential operations, from the definition of coherence tensor in (1), we have

$$
\varepsilon_{j k l} \partial_{k}^{1} E_{l m}+\frac{1}{c} \frac{\partial}{\partial t_{1}} N_{j m}=0
$$

If we apply the same procedure, we obtain

$$
\begin{aligned}
& \varepsilon_{j k l} \partial_{k}^{1} M_{l m}+\frac{1}{c} \frac{\partial}{\partial t_{1}} H_{j m}=0, \\
& \varepsilon_{j k l} \partial_{k}^{1} N_{l m}-\frac{1}{c} \frac{\partial}{\partial t_{1}} E_{j m}=0, \\
& \varepsilon_{j k l} \partial_{k}^{1} H_{l m}-\frac{1}{c} \frac{\partial}{\partial t_{1}} M_{j m}=0 .
\end{aligned}
$$

In a similar manner, the divergence condition yields the following equations:

$$
\begin{aligned}
& \partial_{j}^{1} E_{j k}=0, \\
& \partial_{j}^{1} H_{j k}=0, \\
& \partial_{j}^{1} M_{j k}=0, \\
& \partial_{j}^{1} N_{j k}=0 .
\end{aligned}
$$

After adding (7) to (10), and subtracting (9) from (8), we obtain two equations, respectively:

$$
\begin{aligned}
& \varepsilon_{j k l} \partial_{k}^{1} \mathbb{E}_{l m}-\frac{1}{c} \frac{\partial}{\partial t_{1}} \mathbb{S}_{j m}=0, \\
& \varepsilon_{j k l} \partial_{k}^{1} \mathbb{S}_{l m}+\frac{1}{c} \frac{\partial}{\partial t_{1}} \mathbb{E}_{j m}=0,
\end{aligned}
$$

where

$$
\begin{aligned}
& \mathbb{E}_{j k}\left(\mathbf{x}_{1}, t_{1} ; \mathbf{x}_{2}, t_{2}\right) \\
& =E_{j k}\left(\mathbf{x}_{1}, t_{1} ; \mathbf{x}_{2}, t_{2}\right)+H_{j k}\left(\mathbf{x}_{1}, t_{1} ; \mathbf{x}_{2}, t_{2}\right) \\
& =\operatorname{Tr}\left\{\hat{\rho}\left[\widehat{\mathbf{E}}_{j}^{(-)}\left(\mathbf{x}_{1}, t_{1}\right) \hat{\mathbf{E}}_{k}^{(+)}\left(\mathbf{x}_{2}, t_{2}\right)+\hat{\mathbf{H}}_{j}^{(-)}\left(\mathbf{x}_{1}, t_{1}\right) \hat{\mathbf{H}}_{k}^{(+)}\left(\mathbf{x}_{2}, t_{2}\right)\right]\right\}, \\
& \mathbb{S}_{j k}\left(\mathbf{x}_{1}, t_{1} ; \mathbf{x}_{2}, t_{2}\right) \\
& =M_{j k}\left(\mathbf{x}_{1}, t_{1} ; \mathbf{x}_{2}, t_{2}\right)-N_{j k}\left(\mathbf{x}_{1}, t_{1} ; \mathbf{x}_{2}, t_{2}\right) \\
& =\operatorname{Tr}\left\{\hat{\rho}\left[\widehat{\mathbf{E}}_{j}^{(-)}\left(\mathbf{x}_{1}, t_{1}\right) \hat{\mathbf{H}}_{k}^{(+)}\left(\mathbf{x}_{2}, t_{2}\right)-\hat{\mathbf{H}}_{j}^{(-)}\left(\mathbf{x}_{1}, t_{1}\right) \widehat{\mathbf{E}}_{k}^{(+)}\left(\mathbf{x}_{2}, t_{2}\right)\right]\right\} .
\end{aligned}
$$

The tensor $\mathbb{E}_{j k}$ may be called the quantum energy coherence tensor and $\mathbb{S}_{j k}$ the quantum energy-flow coherence tensor, which have formal resemblance to those of the classical theory [7]. Moreover, from (11) and (12), or from (13) and (14), one has

$$
\begin{aligned}
& \partial_{j}^{1} \mathbb{E}_{j k}=0, \\
& \partial_{j}^{1} \mathbb{S}_{j k}=0 .
\end{aligned}
$$


The equations (15)-(16) and (19)-(20) are basic equations for the propagation of the energy coherence tensor and the energy-flow coherence tensor in free space. Another set of similar equations, which involve $\mathbf{r}_{2}$ and $t_{2}$, can also be derived in a similar way.

So far we have derived the governing equations for the correlations between all the components of the quantum electromagnetic fields at any space-time points. In order to understand the importance of the newly introduced quantum energy and energy-flow coherence tensors, let us discuss the photoelectric detection of the optical field within the quantum mechanical interaction picture. In most of optical experiments, measurements of the electromagnetic field are based on the absorption of photons (energy) via the photoelectric effect. Based on a heuristic argument, Glauber has given the expression for the average counting rate of an ideal photodetection [4], which is proportional to $\operatorname{Tr}\left\{\hat{\rho} \hat{\mathbf{E}}_{j}^{(-)}(\mathbf{x}, t) \hat{\mathbf{E}}_{j}^{(+)}(\mathbf{x}, t)\right\}$. It is important to note here that photon absorption by an ideal detector measures the average value of the Hamiltonian of the quantized radiation field: $\widehat{\mathbf{E}}^{(-)} \cdot \widehat{\mathbf{E}}^{(+)}+\widehat{\mathbf{H}}^{(-)} \cdot \hat{\mathbf{H}}^{(+)}$, and not that of $\widehat{\mathbf{E}}^{(-)} \cdot \widehat{\mathbf{E}}^{(+)}$. One should note that the energies of electric field and magnetic field are indistinguishable in photon absorption process in the detector. Therefore, the probability per unit time that a photon be absorbed by an ideal detector at point $\mathbf{x}$ at time $t$ should be proportional to the expectation value of the normally ordered operator $\widehat{\mathbf{E}}_{j}^{(-)}(\mathbf{x}, t) \widehat{\mathbf{E}}_{j}^{(+)}(\mathbf{x}, t)+$ $\hat{\mathbf{H}}_{j}^{(-)}(\mathbf{x}, t) \hat{\mathbf{H}}_{j}^{(+)}(\mathbf{x}, t)$.

Meanwhile, due to the well-known fact that electromagnetic radiation carries both energy and momentum, any interaction between photons and matters for the exchange of energy will inevitably involve the exchange of momentum. Just as Poynting vector in the classical electromagnetic field, where $\mathbf{S} \propto \mathbf{E}^{*} \times \mathbf{H}+\mathbf{E} \times \mathbf{H}^{*}=\mathbf{E}^{*} \times \mathbf{H}-\mathbf{H}^{*} \times \mathbf{E}$, it is convenient to introduce the notion of energy flow operator, defined by

$$
\widehat{\mathbf{S}}(\mathbf{x}, t)=\widehat{\mathbf{E}}^{(-)}(\mathbf{x}, t) \times \hat{\mathbf{H}}^{(+)}(\mathbf{x}, t)-\hat{\mathbf{H}}^{(-)}(\mathbf{x}, t) \times \widehat{\mathbf{E}}^{(+)}(\mathbf{x}, t) .
$$

The operator $\hat{\mathbf{S}}$ so defined is of course the energy flow associated with the photon absorption in light of the optical equivalence theorem $[8,9]$. Therefore, the average value for the $i$ component of the detected momentum should be proportional to

$$
\operatorname{Tr}\left\{\hat{\rho} \varepsilon_{i j k}\left[\widehat{\mathbf{E}}_{j}^{(-)}(\mathbf{x}, t) \hat{\mathbf{H}}_{k}^{(+)}(\mathbf{x}, t)-\hat{\mathbf{H}}_{j}^{(-)}(\mathbf{x}, t) \widehat{\mathbf{E}}_{k}^{(+)}(\mathbf{x}, t)\right]\right\},
$$

which is the expectation value of the operator $\widehat{\boldsymbol{S}}$ with normal ordering.

Recording the energy and energy flow of photons with a single detector does not exhaust the available measurements we can make upon the field. In the more general expression, the fields $\widehat{\mathbf{E}}^{(-)}, \widehat{\mathbf{E}}^{(+)}, \hat{\mathbf{H}}^{(-)}$, and $\hat{\mathbf{H}}^{(+)}$are evaluated at the different space-time points. Therefore, these tensors $\mathbb{E}_{j k}$ and $\mathbb{S}_{j k}$ furnish a general measure of quantum optical correlations involving all the components of the electromagnetic field at any space-time points and give the field intensity (which is proportional to the counting rate of the detector) and energy flow vector (which is proportional to the kinetic momentum of light) as the special case for $\mathbf{x}_{1}=\mathbf{x}_{2}$ and $t_{1}=t_{2}$. One should be aware of the facts that energy transition is always associated with momentum changes in photon absorption process, and the energies of electric field and magnetic field are indistinguishable in the ideal photodetector. It is the indistinguishability that makes the introduction of $\mathbb{E}_{j k}$ and $\mathbb{S}_{j k}$ meaningful for the better definition of photon-delayed coincidences on the basis of the general first-order coherence tensors.

\section{Coherence Tensor and Vector Potentials}

Borrowing from the general potential theory [10], we can also introduce a new concept of the coherence tensor potential $\mathbb{A}_{l m}$ from which the energy coherence tensor $\mathbb{E}_{j k}$ can be obtained as

$$
\mathbb{E}_{j m}\left(\mathbf{x}_{1}, t_{1} ; \mathbf{x}_{2}, t_{2}\right)=\varepsilon_{j k l} \partial_{k}^{1} \mathbb{A}_{l m}\left(\mathbf{x}_{1}, t_{1} ; \mathbf{x}_{2}, t_{2}\right),
$$

since the divergence of the curl of any tensor is zero, namely,

$$
\begin{aligned}
\partial_{j}^{1}\left(\varepsilon_{j k l} \partial_{k}^{1} \mathbb{A}_{l m}\right) & =\varepsilon_{j k l} \partial_{j}^{1} \partial_{k}^{1} \mathbb{A}_{l m} \\
& =-\varepsilon_{k j l} \partial_{j}^{1} \partial_{k}^{1} \mathbb{A}_{l m} \\
& =-\varepsilon_{j k l} \partial_{j}^{1} \partial_{k}^{1} \mathbb{A}_{l m}=0 .
\end{aligned}
$$

If we substitute from (24) into (16), we have

$$
\varepsilon_{j k l} \partial_{k}^{1}\left(\mathbb{S}_{l m}+\frac{1}{c} \frac{\partial}{\partial t_{1}} \mathbb{A}_{l m}\right)=0 .
$$

Equation (25) will hold if

$$
\begin{aligned}
\mathbb{S}_{l m}\left(\mathbf{x}_{1}, t_{1} ; \mathbf{x}_{2}, t_{2}\right)= & -\frac{1}{c} \frac{\partial}{\partial t_{1}} \mathbb{A}_{l m}\left(\mathbf{x}_{1}, t_{1} ; \mathbf{x}_{2}, t_{2}\right) \\
& -\partial_{l}^{1} \mathbf{a}_{m}\left(\mathbf{x}_{1}, t_{1} ; \mathbf{x}_{2}, t_{2}\right),
\end{aligned}
$$

where $\mathbf{a}_{m}$ is coherence vector potential. $\mathbb{A}_{l m}$ and $\mathbf{a}_{m}$ must now be determined in such a way as to satisfy the remaining Maxwell equations. Let us maintain the following gauge transform relation between $\mathbb{A}_{l m}$ and $\mathbf{a}_{m}$ :

$$
\partial_{l}^{1} \mathbb{A}_{l m}+\frac{1}{c} \frac{\partial}{\partial t_{1}} \mathbf{a}_{m}=0 .
$$

Substituting (26) into (20), and making use of the gauge transform in (27), we can easily find that the coherence vector potential satisfies the wave equation:

$$
\nabla_{1}^{2} \mathbf{a}_{m}-\frac{1}{c^{2}} \frac{\partial^{2}}{\partial t_{1}^{2}} \mathbf{a}_{m}=0 .
$$

Similarly, substituting (23) and (27) into (15), we have

$$
\nabla_{1}^{2} \mathbb{A}_{j m}-\frac{1}{c^{2}} \frac{\partial^{2}}{\partial t_{1}^{2}} \mathbb{A}_{j m}=0,
$$

which indicates the wave equations governing the propagation of the coherence tensor potential. It should be noted 
that the proposed coherence tensor and vector potentials have some similar mathematical behavior with the usual vector and dual potentials [11, 12], but the underlying physics is completely different. The key point of the proposed coherence potentials is to govern propagation of quantum correlation, whereas the usual vector and dual potentials are developed for the description of the electromagnetic wave in nonlinear dielectric media. The significance of the coherence tensor and vector potentials can be explained as follows. Just as the vector and scalar potentials play a more fundamental role in quantum electrodynamics than the electromagnetic fields themselves [13], the newly introduced coherence tensor potential and coherence vector potential are expected to play a more fundamental role in the descriptions of the quantum correlation and entanglement than the energy coherence tensor and the energy-flow coherence tensor.

\section{Conservation Law of Quantum-Correlation-Function Energy}

Noting the formal analogy to Maxwell's equations in free space, we can introduce some concepts to characterize the spatiotemporal evolution of the energy coherence tensor and the energy-flow coherence tensor. Borrowed from scalar theory in classical wavefields, an energy-flow-density-like quantity has been defined and observed experimentally when a generic coherence vortex is reported $[14,15]$. Under the framework of classical scalar coherence theory, the associated conservation law has been deduced. However, with the optical equivalence theorem for normally order operators $[8,9]$ as a clue, it is possible to extend this concept so as to take into account the vectorial character of light to formulate much rigorous laws for quantum optics.

Let us now take the complex conjugate of (15) and multiply by $\mathbb{S}_{j m}$. We then obtain

$$
\varepsilon_{j k l} \mathbb{S}_{j m} \partial_{k}^{1} \mathbb{E}_{l m}^{*}-\frac{1}{c} \mathbb{S}_{j m} \frac{\partial}{\partial t_{1}} \mathbb{S}_{j m}^{*}=0 .
$$

If we add (30) with its complex conjugate, we have

$$
\frac{1}{c} \frac{\partial}{\partial t_{1}}\left(\mathbb{S}_{j m}^{*} \mathbb{S}_{j m}\right)-\varepsilon_{j k l}\left(\mathbb{S}_{j m}^{*} \partial_{k}^{1} \mathbb{E}_{l m}+\mathbb{S}_{j m} \partial_{k}^{1} \mathbb{E}_{l m}^{*}\right)=0
$$

If we make use of relation $\varepsilon_{j k l}=-\varepsilon_{l k j}$ and interchange the dummy suffices $j$ and $m$, we have

$$
\frac{1}{c} \frac{\partial}{\partial t_{1}}\left(\mathbb{S}_{j m}^{*} \mathbb{S}_{j m}\right)+\varepsilon_{j k l}\left(\mathbb{S}_{l m}^{*} \partial_{k}^{1} \mathbb{E}_{j m}+\mathbb{S}_{l m} \partial_{k}^{1} \mathbb{E}_{j m}^{*}\right)=0
$$

In a similar way, we obtain from (16)

$$
\frac{1}{c} \frac{\partial}{\partial t_{1}}\left(\mathbb{E}_{j m}^{*} \mathbb{E}_{j m}\right)+\varepsilon_{j k l}\left(\mathbb{E}_{j m}^{*} \partial_{k}^{1} \mathbb{S}_{l m}+\mathbb{E}_{j m} \partial_{k}^{1} \mathbb{S}_{l m}^{*}\right)=0 .
$$

After adding (32) to (33), we readily obtain

$$
\frac{\partial}{\partial t_{1}} W\left(\mathbf{x}_{1}, t_{1} ; \mathbf{x}_{2}, t_{2}\right)+\partial_{k}^{1} \mathbf{T}_{k}\left(\mathbf{x}_{1}, t_{1} ; \mathbf{x}_{2}, t_{2}\right)=0,
$$

where

$$
\begin{gathered}
W\left(\mathbf{x}_{1}, t_{1} ; \mathbf{x}_{2}, t_{2}\right)=\mathbb{E}_{j m}^{*} \mathbb{E}_{j m}+\mathbb{S}_{j m}^{*} \mathbb{S}_{j m}, \\
\mathbf{T}_{\mathrm{k}}\left(\mathbf{x}_{1}, t_{1} ; \mathbf{x}_{2}, t_{2}\right)=c \varepsilon_{k l j}\left(\mathbb{S}_{l m} \mathbb{E}_{j m}^{*}+\mathbb{S}_{l m}^{*} \mathbb{E}_{j m}\right) .
\end{gathered}
$$

Apart from a constant factor, (34) has a formal resemblance to that of the classical theory (see [7, Equation (3.5)]), while the physical meaning has received much less attention in the latter. From its formal analogy to energy conservation law in electromagnetism, we note that (34) is a continuity equation in which the scalar quantity $W\left(\mathbf{x}_{1}, t_{1} ; \mathbf{x}_{2}, t_{2}\right)$ may be regarded as representing an energy-density-like quantity of quantum correlation (which we term the quantum-correlation-function energy density) and the vector quantity $\mathbf{T}\left(\mathbf{x}_{1}, t_{1} ; \mathbf{x}_{2}, t_{2}\right)$ as representing a flow-density-like quantity of quantum correlation (which we term the quantum-correlation-function flow density). Just as the magnitude and the direction of the Poynting vector represent, respectively, the field intensity and the direction of light energy flow in classic optics, the magnitude and the direction of the quantum-correlationfunction flow density vector $\mathbf{T}\left(\mathbf{x}_{1}, t_{1} ; \mathbf{x}_{2}, t_{2}\right)$ give, respectively, a measure of the intensity of quantum correlation and the direction of the quantum correlation flow. If we integrate (34) throughout a volume $V$ bounded by a closed surface $S$ and apply Gauss' theorem, we have

$$
\begin{aligned}
& \frac{\mathrm{d}}{\mathrm{d} t_{1}} \iiint_{V} W\left(\mathbf{x}_{1}, t_{1} ; \mathbf{x}_{2}, t_{2}\right) \mathrm{d}^{3} x_{1} \\
& \quad+\iint_{S} \mathbf{T}\left(\mathbf{x}_{1}, t_{1} ; \mathbf{x}_{2}, t_{2}\right) \cdot \mathbf{n d}^{2} x_{1}=0
\end{aligned}
$$

where $\mathbf{n}$ denotes the unit outward normal to $S$. Equation (37) may be given the following interpretation. The rate of increase (or decrease) of the quantum-correlation-function energy contained in $V$ is equal to the rate at which the quantum-correlation-function energy enters (or leaves) $V$ through the boundary $S$ via the quantum-correlationfunction flow. With this interpretation (34) expresses the conservation law of quantum-correlation-function energy, which may be regarded as natural generalizations of those obtained on the basis of classical scalar coherence theory [15].

\section{Conservation Law of Quantum-Correlation-Function Momemtum}

Now, let us consider a new law on the conservation of a momentum-like quantity in quantum correlation theory. If we take the complex conjugate of (15) and multiply by $\varepsilon_{i n j} \mathbb{E}_{n m}$ and make use of tensor identity $\varepsilon_{j k l} \varepsilon_{i n j}=\varepsilon_{k l j} \varepsilon_{i n j}=$ $\delta_{k i} \delta_{l n}-\delta_{k n} \delta_{l i}$ (where $\delta_{k i}$ is equal to zero if $k \neq i$, and equal to unity if $k=i$ ), we then obtain

$$
-\mathbb{E}_{n m} \partial_{i}^{1} \mathbb{E}_{n m}^{*}+\mathbb{E}_{n m} \partial_{n}^{1} \mathbb{E}_{i m}^{*}+\mathbb{E}_{i m}^{*} \partial_{n}^{1} \mathbb{E}_{n m}+\frac{1}{c} \varepsilon_{i n j} \mathbb{E}_{n m} \frac{\partial}{\partial t_{1}} \mathbb{S}_{j m}^{*}=0,
$$


where the use has been made of the divergence-free condition in (19). Similarly, if we apply the similar operation to (16) and use the tensor identity, we have

$$
-\mathbb{S}_{n m} \partial_{i}^{1} \mathbb{S}_{n m}^{*}+\mathbb{S}_{n m} \partial_{n}^{1} \mathbb{S}_{i m}^{*}+\mathbb{S}_{i m}^{*} \partial_{n}^{1} \mathbb{S}_{n m}+\frac{1}{c} \varepsilon_{i n j} \mathbb{S}_{j m} \frac{\partial}{\partial t_{1}} \mathbb{E}_{n m}^{*}=0,
$$

where the use has also been made of the divergence-free condition in (20). Let us add (38) and its complex conjugate to (39) and its complex conjugate; we finally arrive at

$$
\frac{\partial}{\partial t_{1}} \mathbb{T}_{i}\left(\mathbf{x}_{1}, t_{1} ; \mathbf{x}_{2}, t_{2}\right)+\partial_{k}^{1} \mathbb{W}_{k i}\left(\mathbf{x}_{1}, t_{1} ; \mathbf{x}_{2}, t_{2}\right)=0
$$

where

$$
\begin{aligned}
\mathbb{T}_{i}= & \frac{\mathbf{T}_{i}}{c^{2}}=\frac{1}{c} \varepsilon_{i k j}\left(\mathbb{S}_{k m} \mathbb{E}_{j m}^{*}+\mathbb{S}_{k m}^{*} \mathbb{E}_{j m}\right), \\
\mathbb{W}_{k i}= & \left(\mathbb{E}_{k m} \mathbb{E}_{i m}^{*}+\mathbb{E}_{k m}^{*} \mathbb{E}_{i m}\right)-\delta_{k i}\left(\mathbb{E}_{k m} \mathbb{E}_{k m}^{*}\right) \\
& +\left(\mathbb{S}_{k m} \mathbb{S}_{i m}^{*}+\mathbb{S}_{k m}^{*} \mathbb{S}_{i m}\right)-\delta_{k i}\left(\mathbb{S}_{k m} \mathbb{S}_{k m}^{*}\right) .
\end{aligned}
$$

If we integrate (40) throughout the volume $V$ bounded by a closed surface $S$ and apply Gauss' theorem in the tensor form, we have

$$
\begin{aligned}
& \frac{\mathrm{d}}{\mathrm{d} t_{1}} \iiint_{V} \mathbb{T}_{i}\left(\mathbf{x}_{1}, t_{1} ; \mathbf{x}_{2}, t_{2}\right) \mathrm{d}^{3} x_{1} \\
& \quad+\iint_{S} \mathbb{W}_{k i}\left(\mathbf{x}_{1}, t_{1} ; \mathbf{x}_{2}, t_{2}\right) n_{k} \mathrm{~d}^{2} x_{1}=0,
\end{aligned}
$$

where $n_{k}$ is the $k$ component of unit outward normal vector n. From its formal analogy to momentum conservation law in electromagnetism, we interpret the vector $\mathbb{T}\left(\mathbf{x}_{1}, t_{1} ; \mathbf{x}_{2}, t_{2}\right)$ as a momentum-density-like quantity of quantum correlation (which we term the quantum-correlation-function momentum density). Furthermore, the symmetric tensor $W_{k i}\left(\mathbf{x}_{1}, t_{1} ; \mathbf{x}_{2}, t_{2}\right)$ may be regarded as a Maxwell-stress-tensorlike quantity of quantum correlation (which we term the quantum-correlation-function stress tensor), representing the flux of the $k$ th component of the quantum-correlationfunction momentum in the $i$ th direction. Hence, the above equation states that the rate of gain (or loss) of quantumcorrelation-function momentum in the closed volume is equal to the flux of quantum-correlation-function momentum flowing into (or out of) the volume across the bounding surface. With this interpretation, (40) expresses the conservation law of quantum-correlation-function momentum, which bears a striking resemblance to the classical one [16, 17].

\section{Relation between Energy and Momentum in Quantum Correlation Function}

Borrowing from the classical optics [18], we can introduce some geometrical concepts to represent the quantumcorrelation-function flow. Let us consider the propagation of the quantum correlation function with a speed $c$ through an area $S$ normal to the direction of propagation. During a very small interval of time $\Delta t$, only the quantumcorrelation-function energy contained in the cylindrical volume, $(c \Delta t S) W$, will flow across $S$. We now make the reasonable assumption (for vacuum) that the quantumcorrelation-function flow is in the direction of propagation of the quantum correlation. The magnitude of the corresponding quantum-correlation-function flow is then

$$
|\mathbf{T}|=\frac{c \Delta t S W}{\Delta t S}=c W .
$$

Since $\mathbb{T} c^{2}=\mathbf{T}$ (in (41)), we can express the quantumcorrelation-function energy in terms of the magnitude of the quantum-correlation-function momentum, namely,

$$
W=c|\mathbb{T}| \text {. }
$$

Notice, from (45), that if some amount of quantumcorrelation-function energy $W$ is transported per square meter per second, then there will be a corresponding quantum-correlation-function momentum $W / c$ transported per square meter per second. Thus, the mathematical relation between the energy and momentum in quantum correlation function is essentially identical to the energy- momentum relation in classical electrodynamics.

\section{Conservation of Quantum-Correlation-Function Angular Momentum}

Finally, we remark that the quantum-correlation-function angular momentum in quantum theory of optical coherence can also be treated in a similar way. The angular momentum density associated with the correlation tensors of the quantized field is given by

$$
\mathbb{L}_{m}\left(\mathbf{x}_{1}, t_{1} ; \mathbf{x}_{2}, t_{2}\right)=\varepsilon_{m j i} x_{j} \mathbb{T}_{i}\left(\mathbf{x}_{1}, t_{1} ; \mathbf{x}_{2}, t_{2}\right),
$$

where $\mathbb{T}_{i}$ is the quantum-correlation-function momentum density discussed above. The flux of angular momentum density in quantum correlation function is given by

$$
\mathbb{M}_{m k}\left(\mathbf{x}_{1}, t_{1} ; \mathbf{x}_{2}, t_{2}\right)=\varepsilon_{m j i} x_{j} \mathbb{W}_{i k}\left(\mathbf{x}_{1}, t_{1} ; \mathbf{x}_{2}, t_{2}\right),
$$

where $\mathbb{W}_{k i}$ is the quantum-correlation-function stress tensor given above. Conservation of angular momentum in quantum correlation function is also expressed by

$$
\frac{\partial}{\partial t_{1}} \mathbb{L}_{m}\left(\mathbf{x}_{1}, t_{1} ; \mathbf{x}_{2}, t_{2}\right)+\partial_{k}^{1} \mathbb{M}_{m k}\left(\mathbf{x}_{1}, t_{1} ; \mathbf{x}_{2}, t_{2}\right)=0,
$$

which again bears the close analogy to the classical theories of coherence [17].

\section{Specific Examples and Physical Interpretations}

So far, on the basis of the theory of the quantized electromagnetic field, we have introduced some vector and tensor 
densities to the quantum theory of optical coherence and have developed a very close analogy between the quantum mechanical and classical theories of coherence. To offer much more penetrating insights into the role played by quantumcorrelation-function conservations in the description of the coherence properties of the quantized field, we will carry out mathematical development through the use of some particular sets of quantum states. Without loss of generality, the expansion for the vector potential of the quantized multimode electromagnetic field with its linear polarization along the $\widehat{\mathbf{x}}$-direction takes the form

$$
\begin{aligned}
\hat{\mathbf{A}}_{x}(z, t)= & \frac{c}{L^{3 / 2}} \sum_{m}\left[\frac{\hbar}{\left(2 \omega_{m}\right)}\right]^{1 / 2} \\
& \times\left\{\hat{a}_{m} \exp \left[i \omega_{m}\left(\frac{z}{c}-t\right)\right]+\text { h.c. }\right\},
\end{aligned}
$$

where $\omega_{m}$ is the angular frequency of the $m$ th mode, $L$ is the side length of a cubical volume, and h.c. stands for the Hermitian conjugate of the preceding term. From the expansions for the vector potential $\hat{\mathbf{A}}$ of the electromagnetic filed, the electric and magnetic field operators $\hat{\mathbf{E}}$ and $\hat{\mathbf{H}}$ can therefore be written as

$$
\begin{aligned}
\widehat{\mathbf{E}}_{x}(z, t)= & \hat{\mathbf{H}}_{y}(z, t) \\
= & \frac{i}{L^{3 / 2}} \sum_{m}\left(\frac{\hbar \omega_{m}}{2}\right)^{1 / 2} \\
& \times\left\{\hat{a}_{m} \exp \left[i \omega_{m}\left(\frac{z}{c}-t\right)\right]+\text { h.c. }\right\} .
\end{aligned}
$$

By inserting the operators of the quantized fields $\hat{\mathbf{E}}$ and $\hat{\mathbf{H}}$ into the definition for energy coherence tensor $\mathbb{E}_{j k}$ and the energy-flow coherence tensor $\mathbb{S}_{j k}$, we can evaluate the proposed quantum-correlation-function energy $W$ and quantum-correlation-function momentum $\mathbb{T}$ at some particular set of quantum states of the field.

8.1. Quantum Correlation Function in Fock States. As a first example, consider a multimode of the electromagnetic field in an eigenstate of Fock states (photon number states). After calculating the E-only correlation tensor, $\mathbf{H}$-only correlation tensor, and EH mixed-correlation tensors from (1), we have

$$
\begin{aligned}
\mathrm{E}_{x x} & =\mathrm{H}_{y y}=M_{x y}=N_{y x} \\
& =\frac{\hbar}{2 L^{3}} \sum_{m}\left\{\omega_{m}\left\langle n_{m}\right\rangle \exp \left[i \omega_{m}\left(\frac{z_{2}}{c}-t_{2}-\frac{z_{1}}{c}+t_{1}\right)\right]\right\} \\
& =\frac{\hbar}{2 L^{3}} \sum_{m} \phi_{m},
\end{aligned}
$$

where $\operatorname{Tr}\left\{\hat{\rho} \hat{a}_{m}^{\dagger} \hat{a}_{m}\right\}=\left\langle n_{m}\right\rangle$ is the average photon number of the $m$ th mode, and we have put for short $\phi_{m}=$ $\omega_{m}\left\langle n_{m}\right\rangle \exp \left[i \omega_{m}\left(z_{2} / c-t_{2}-z_{1} / c+t_{1}\right)\right]$. Substituting from $(51)$ into (17)-(18), we obtain

$$
\begin{aligned}
& \mathbb{E}=\left(\begin{array}{ccc}
\frac{\hbar}{2 L^{3}} \sum_{m} \phi_{m} & 0 & 0 \\
0 & \frac{\hbar}{2 L^{3}} \sum_{m} \phi_{m} & 0 \\
0 & 0 & 0
\end{array}\right), \\
& \mathbb{S}=\left(\begin{array}{ccc}
0 & \frac{\hbar}{2 L^{3}} \sum_{m} \phi_{m} & 0 \\
-\frac{\hbar}{2 L^{3}} \sum_{m} \phi_{m} & 0 & 0 \\
0 & 0 & 0
\end{array}\right) .
\end{aligned}
$$

Apart from a constant factor, the elements of the energy and energy flow coherence tensors are represented as the sum of many elementary phasor contributions $\phi_{m}$. Thus, the density of quantum-correlation-function energy is proportional to the square of the absolute value of this sum. After substituting (52)-(53) into (35), we have

$$
W=4\left(\frac{\hbar}{2 L^{3}} \sum_{m} \phi_{m}\right)\left(\frac{\hbar}{2 L^{3}} \sum_{m} \phi_{m}\right)^{*}=\frac{\hbar^{2}}{L^{6}}\left|\sum_{m} \phi_{m}\right|^{2} .
$$

Equation (54) evidently introduces the phenomenon of interference into the scheme of the quantum theory of optical coherence. After straightforward algebra, the quantumcorrelation-function energy is readily shown to be

$$
\begin{aligned}
W=\frac{\hbar^{2}}{L^{6}}\left\{\sum_{m}\right. & \omega_{m}^{2}\left\langle n_{m}\right\rangle^{2}+2 \sum_{m} \sum_{l \neq m} \omega_{m} \omega_{l}\left\langle n_{m}\right\rangle\left\langle n_{l}\right\rangle \\
& \left.\times \cos \left[\left(\omega_{m}-\omega_{l}\right)\left(z_{2} / c-t_{2}-z_{1} / c+t_{1}\right)\right]\right\} .
\end{aligned}
$$

It is immediately seen from (55) that the density of quantumcorrelation-function energy will depend on the two spatiotemporal variables only through their difference and has both particle and wave manifestations. For the single-point correlation function, where $z_{1}=z_{2}, t_{1}=t_{2}$, we have

$$
W=\frac{1}{L^{6}}\left[\sum_{m}\left(\left\langle n_{m}\right\rangle \hbar \omega_{m}\right)\right]^{2} .
$$

Thus the quantum-correlation-function energy density is discrete with particle behavior. Meanwhile, the quantumcorrelation-function energy density has uniform distribution for all the spatiotemporal position. Meanwhile, the quantum-correlation-function energy density exhibits wavelike features for the two-point correlation in photon delayed coincidence. With this interpretation, (55) expresses the simultaneous wave-particle duality of quantum-correlationfunction energy density. The dynamical behavior of the quantum correlation is governed by the total quantumcorrelation-function energy that takes the form

$$
\begin{aligned}
\mathcal{W} & =\int_{V} W\left(\mathbf{x}_{1}, t_{1} ; \mathbf{x}_{2}, t_{2}\right) \mathrm{d}^{3} x_{1} \\
& =\frac{1}{L^{3}} \sum_{m} \hbar^{2} \omega_{m}^{2}\left\langle n_{m}\right\rangle^{2} .
\end{aligned}
$$


Similarly, with the use of (52)-(53) for the expressions of the quantum energy and energy flow coherence tensor, the quantum-correlation-function momentum vector density has only $\hat{\mathbf{z}}$-direction component:

$$
\begin{aligned}
\mathbb{T}_{z}=\frac{\hbar^{2}}{c L^{6}}\left\{\sum_{m} \omega_{m}^{2}\left\langle n_{m}\right\rangle^{2}+2 \sum_{m} \sum_{l \neq m} \omega_{m} \omega_{l}\left\langle n_{m}\right\rangle\left\langle n_{l}\right\rangle\right. \\
\left.\quad \times \cos \left[\left(\omega_{m}-\omega_{l}\right)\left(\frac{z_{2}}{c}-t_{2}-\frac{z_{1}}{c}+t_{1}\right)\right]\right\} .
\end{aligned}
$$

As expected, the quantum-correlation-function momentum also exhibits the wave-particle duality. Then, the total quantum-correlation function momentum within the cubical volume is

$$
\mathcal{T}_{z}=\int_{V} \mathbb{T}_{z}\left(\mathbf{x}_{1}, t_{1} ; \mathbf{x}_{2}, t_{2}\right) \mathrm{d}^{3} x_{1}=\frac{1}{c L^{3}} \sum_{m} \hbar^{2} \omega_{m}^{2}\left\langle n_{m}\right\rangle^{2} .
$$

From the formal analogy to the concept of single photon in quantum optics, we may envision the quantum correlation of a single mode carrying a discrete quantumcorrelation-function energy $\mathcal{W}=\langle n\rangle^{2} \hbar^{2} \omega^{2} / L^{3}$ with a quantum-correlation-function momentum $\mathcal{T}_{z}=W / c=$ $\langle n\rangle^{2} \hbar^{2} \omega^{2} /\left(c L^{3}\right)$.

In light of the wave-particle duality of quantum physics, it is natural for the photon to carry the properties of both waves and of particles. Owing the Wolf [19], it has been known since 1955 that the optical coherence exhibits wave properties since the coherence function obeys a couple of wave equations. Here, it is interesting to note that, besides wave properties, the quantum correlation function also has particle nature since the correlations between the discrete photons (photon correlations) play the essential role in the quantum theory of optical coherence.

8.2. Quantum Correlation in Coherent States. In quantum optics, it is also common practice to describe the state of the photon field in terms of coherent states $[4,5]$. As stressed by Glauber, one of the important properties for the coherent states is that the correlation functions can be factorized if the field is in coherent state. Therefore, the first-order correlation functions reduce to the factorized forms:

$$
\begin{aligned}
E_{j k}\left(\mathbf{x}_{1}, t_{1} ; \mathbf{x}_{2}, t_{2}\right) & =\mathcal{E}_{j}^{*}\left(\mathbf{x}_{1}, t_{1}\right) \mathscr{E}_{k}\left(\mathbf{x}_{2}, t_{2}\right), \\
H_{j k}\left(\mathbf{x}_{1}, t_{1} ; \mathbf{x}_{2}, t_{2}\right) & =\mathscr{H}_{j}^{*}\left(\mathbf{x}_{1}, t_{1}\right) \mathscr{H}_{k}\left(\mathbf{x}_{2}, t_{2}\right), \\
M_{j k}\left(\mathbf{x}_{1}, t_{1} ; \mathbf{x}_{2}, t_{2}\right) & =\mathcal{E}_{j}^{*}\left(\mathbf{x}_{1}, t_{1}\right) \mathscr{H}_{k}\left(\mathbf{x}_{2}, t_{2}\right), \\
N_{j k}\left(\mathbf{x}_{1}, t_{1} ; \mathbf{x}_{2}, t_{2}\right) & =\mathscr{H}_{j}^{*}\left(\mathbf{x}_{1}, t_{1}\right) \mathcal{E}_{k}\left(\mathbf{x}_{2}, t_{2}\right),
\end{aligned}
$$

in which the functions $\mathscr{E}(\mathbf{x}, t)$, and $\mathscr{H}(\mathbf{x}, t)$ and their corresponding complex conjugates play the role of the eigenvalue and satisfy the Maxwell equations. According to (50), the eigenvalue functions $\mathcal{E}_{j}(\mathbf{x}, t)$ and $\mathscr{H}_{j}(\mathbf{x}, t)$ are thus given by

$$
\begin{aligned}
\mathcal{E}_{x}(z, t) & =\mathscr{H}_{y}(z, t) \\
& =\frac{i}{L^{3 / 2}} \sum_{m}\left(\frac{\hbar \omega_{m}}{2}\right)^{1 / 2} \alpha_{m} \exp \left[i \omega_{m}\left(\frac{z}{c}-t\right)\right],
\end{aligned}
$$

where $\alpha_{m}$ is eigenvalue of the annihilation operator $\hat{a}_{m}$, that is, $\hat{a}_{m}\left|\alpha_{m}\right\rangle=\alpha_{m}\left|\alpha_{m}\right\rangle$. As we readily see, the energy and energy flow coherence tensors of the quantized field satisfy

$$
\begin{aligned}
& \mathbb{E}=\left(\begin{array}{ccc}
\mathcal{E}_{x}^{*}\left(z_{1}, t_{1}\right) \mathcal{E}_{x}\left(z_{2}, t_{2}\right) & 0 & 0 \\
0 & \mathcal{H}_{y}^{*}\left(z_{1}, t_{1}\right) \mathscr{H}_{y}\left(z_{2}, t_{2}\right) & 0 \\
0 & 0 & 0
\end{array}\right), \\
& \mathbb{S}=\left(\begin{array}{ccc}
-\mathscr{H}_{y}^{*}\left(z_{1}, t_{1}\right) \mathcal{E}_{x}\left(z_{2}, t_{2}\right) & \mathcal{E}_{x}^{*}\left(z_{1}, t_{1}\right) \mathscr{H}_{y}\left(z_{2}, t_{2}\right) & 0 \\
0 & 0 & 0 \\
0 & 0 & 0
\end{array}\right) .
\end{aligned}
$$

Using the results of (62), we find that

$$
\begin{aligned}
W= & {\left[\left|\mathcal{E}_{x}\left(z_{1}, t_{1}\right)\right|^{2}+\left|\mathcal{H}_{y}\left(z_{1}, t_{1}\right)\right|^{2}\right] } \\
& \times\left[\left|\mathcal{E}_{x}\left(z_{2}, t_{2}\right)\right|^{2}+\left|\mathcal{H}_{y}\left(z_{2}, t_{2}\right)\right|^{2}\right] \\
= & 4\left|\mathcal{E}_{x}\left(z_{1}, t_{1}\right)\right|^{2} \cdot\left|\mathcal{E}_{x}\left(z_{2}, t_{2}\right)\right|^{2} .
\end{aligned}
$$

Similarly, the quantum-correlation-function momentum density takes the form

$$
\begin{aligned}
\mathbb{T}_{z}= & 2 c^{-1} \operatorname{Re}\left\{\mathcal{E}_{x}^{*}\left(z_{1}, t_{1}\right) \mathcal{H}_{y}\left(z_{1}, t_{1}\right)\right\} \\
& \times\left[\left|\mathcal{E}_{x}\left(z_{2}, t_{2}\right)\right|^{2}+\left|\mathcal{H}_{y}\left(z_{2}, t_{2}\right)\right|^{2}\right] \\
= & 4 c^{-1}\left|\mathcal{E}_{x}\left(z_{1}, t_{1}\right)\right|^{2} \cdot\left|\mathcal{E}_{x}\left(z_{2}, t_{2}\right)\right|^{2}=\frac{W}{c},
\end{aligned}
$$

where $\operatorname{Re}\{\cdots\}$ stands for the real part of the complex eigenvalue. After substituting (61) into (63)-(64), we see that the coherent state of a single mode has quantum-correlationfunction energy $\mathcal{W}=(\hbar \omega)^{2}|\alpha|^{4} / L^{3}$ with quantumcorrelation-function momentum $\mathcal{T}_{z}=c(\hbar k)^{2}|\alpha|^{4} / L^{3}$.

Up to now we have considered the conservation laws in the quantum correlation function based on the correlation tensors of first-order only. It should also be noted that the generalization to higher-order correlation tensors can also be derived in a similar way.

Meanwhile, the proposed conservation laws in quantum correlation function may also provide an insight into other correlation phenomena in quantum mechanics. For example, the Einstein-Podolsky-Rosen (EPR) paradox draws on a phenomenon predicted by quantum mechanics, known as quantum entanglement, to show that measurements performed on spatially separated parts of a quantum system can apparently have an instantaneous influence on one another [20]. Since photons are emitted from a common source, correlations between these photons have already been established once an entangled state is created [21, 22]. In an entangled two-photon state, a measurement of one chosen variable of photon one will determine the outcome of a measurement of the corresponding variable of the other photons. No matter how far apart for these two photons, the proposed conservation laws always hold for this quantum system. It is these conservation laws for quantum correlation function that add an additional condition for 
the propagation of the separated photons in their entangled state when no influence resulting from one measurement can possibly propagate to the other photon in the available time. This may give the origin of the nonlocal behavior or spooky action at a distance for quantum mechanics.

\section{Conclusion}

In summary, we have derived the expressions for energy coherence tensor and the energy-flow coherence tensor on the basis of quantum field theory, which provides new insights into photon statistics and quantum correlation. In terms of a electromagnetic model, we have introduced new quantities to quantum theory of optical coherence based on newly defined quantum optical coherence tensors and have related them by continuity equations, giving new conservation laws in quantum correlation function. Furthermore, we have theoretically investigated the propagation of quantum correlation function, which establishes a new relationship between the photon correlation and quantum electrodynamics and reveals the wave-particle duality of quantum correlation. Further exploration in this direction may lead to a new field in quantum optics that may be referred to as Quantum Correlation Dynamics.

\section{Acknowledgments}

The authors are indebted to Zhengwei Zhou for his helpful discussion. Part of this work was supported by Grant-inAid of JSPS B no. 21360028 and by The 21st Century Center of Excellence (COE) Program on "Innovation of Coherent Optical Science" granted to The University of Electro-Communications.

\section{References}

[1] L. Mandel and E. Wolf, Optical Coherence and Quantum Optics, Cambridge University Press, Cambridge, UK, 1995.

[2] J. Perina, Quantum Statistics of Linear and Nonlinear Optical Phenomena, Kluwer Acadmic, Dordrecht, The Netherlands, 1991.

[3] R. J. Glauber, "Photon correlations," Physical Review Letters, vol. 10, pp. 84-86, 1963.

[4] R. J. Glauber, "The quantum theory of optical coherence," Physical Review, vol. 130, no. 6, pp. 2529-2539, 1963.

[5] R. J. Glauber, "Coherent and incoherent states of the radiation field," Physical Review, vol. 131, no. 6, pp. 2766-2788, 1963.

[6] C. L. Mehta and E. Wolf, "Coherence properties of blackbody radiation. II. Correlation tensors of the quantized field," Physical Review, vol. 134, no. 5A, pp. A1149-A1153, 1964.

[7] P. Roman and E. Wolf, "Correlation theory of stationary electromagnetic fields-part II: conservation laws," Il Nuovo Cimento, vol. 17, no. 4, pp. 477-490, 1960.

[8] E. C. G. Sudarshan, "Equivalence of semiclassical and quantum mechanical descriptions of statistical light beams," Physical Review Letters, vol. 10, no. 7, pp. 277-279, 1963.

[9] J. R. Klauder, "Improved version of optical equivalence theorem," Physical Review Letters, vol. 16, no. 12, pp. 534-536, 1966.

[10] O. D. Kellogg, Foundations of Potential Theory, Dover, New York, NY, USA, 1954.
[11] M. Hillery and L. D. Mlodinow, "Quantization of electrodynamics in nonlinear dielectric media," Physical Review A, vol. 30, no. 4, pp. 1860-1865, 1984.

[12] S. Serulnik and Y. Ben-Aryeh, "Space-time description of propagation in nonlinear dielectric media," Quantum Optics, vol. 3, no. 1, pp. 63-74, 1991.

[13] Y. Aharonov and D. Bohm, "Significance of electromagnetic potentials in the quantum theory," Physical Review, vol. 115, no. 3, pp. 485-491, 1959.

[14] W. Wang, Z. Duan, S. G. Hanson, Y. Miyamoto, and M. Takeda, "Experimental study of coherence vortices: local properties of phase singularities in a spatial coherence function," Physical Review Letters, vol. 96, no. 7, Article ID 073902, 4 pages, 2006.

[15] W. Wang and M. Takeda, "Coherence current, coherence vortex, and the conservation law of coherence," Physical Review Letters, vol. 96, no. 22, Article ID 223904, 4 pages, 2006.

[16] P. Roman, "Correlation theory of stationary electromagnetic fields. Part IV. Second order conservation laws," Il Nuovo Cimento, vol. 22, no. 5, pp. 1005-1011, 1961.

[17] W. Wang and M. Takeda, "Linear and angular coherence momenta in the classical second-order coherence theory of vector electromagnetic fields," Optics Letters, vol. 31, no. 17, pp. 2520-2522, 2006.

[18] E. Hecht, Optics, Addison Wesley, Boston, Mass, USA, 2001.

[19] E. Wolf, "A macroscopic theory of interference and diffraction of light from finite sources. II. Fields with a spectral range of arbitrary width," Proceedings of the Royal Society A, vol. 230, no. 1181, pp. 246-265, 1955.

[20] A. Einstein, B. Podolsky, and N. Rosen, "Can quantummechanical description of physical reality be considered complete?" Physical Review, vol. 47, no. 10, pp. 777-780, 1935.

[21] D. Bohm, "A suggested interpretation of the quantum theory in terms of "hidden" variables. I," Physical Review, vol. 85, no. 2, pp. 166-179, 1952.

[22] D. Bohm, "A suggested interpretation of the quantum theory in terms of "hidden" variables. II," Physical Review, vol. 85, no. 2, pp. 180-193, 1952. 

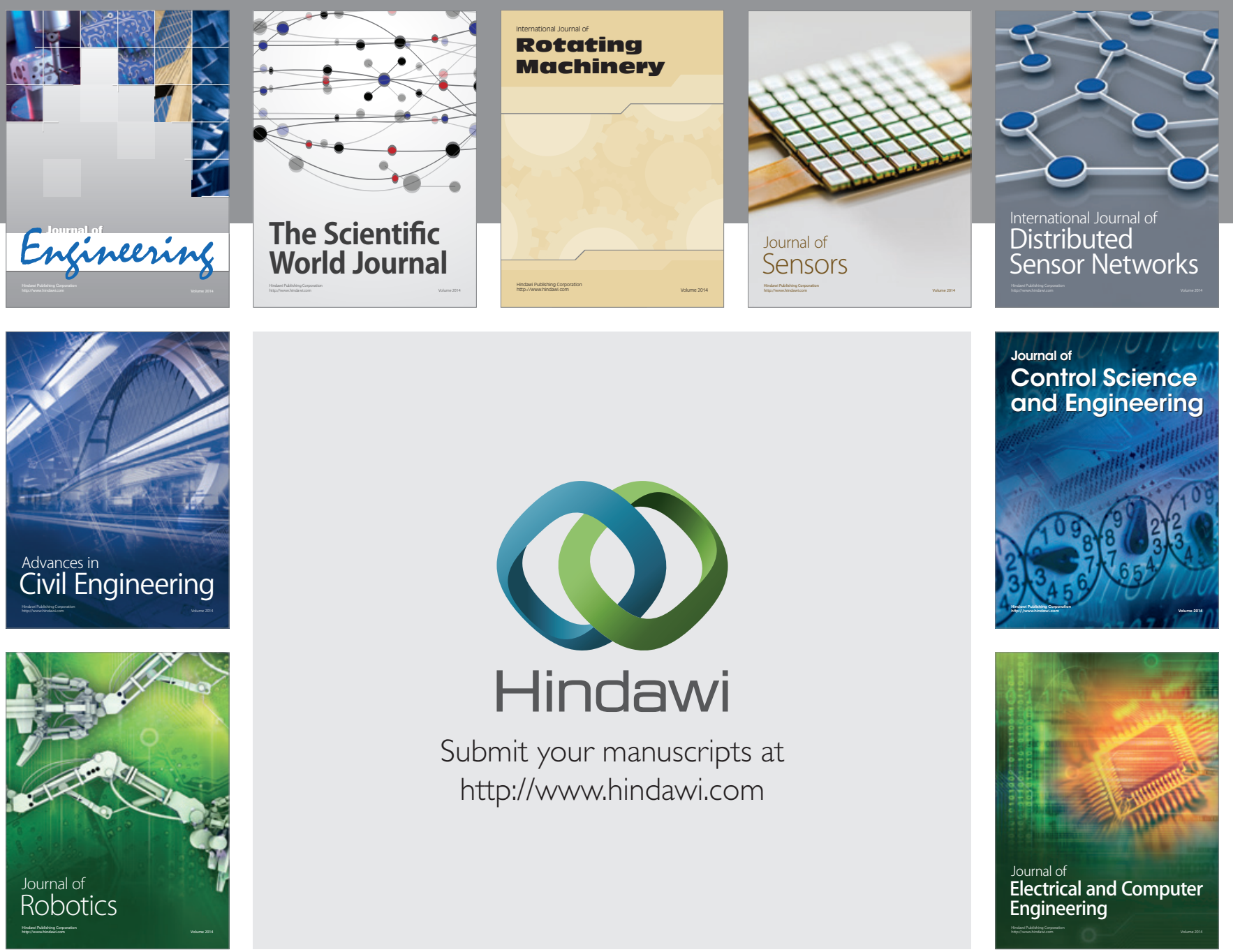

Submit your manuscripts at

http://www.hindawi.com
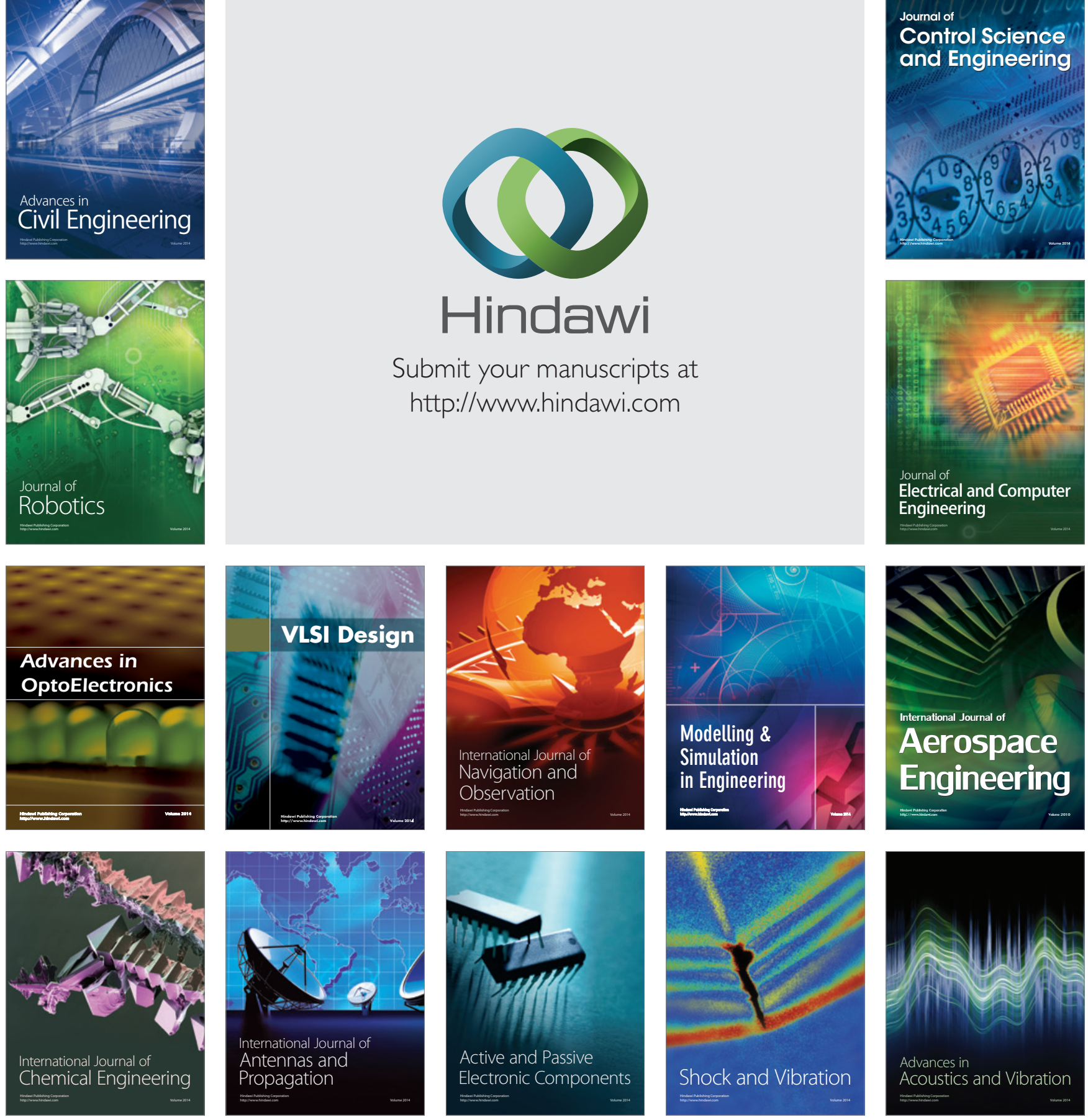